\title{
A computational study of biomagnetic fluid flow in a channel in the presence of obstacles under the influence of the magnetic field generated by a wire carrying electric current
}

\section{S. Morteza Mousavi, Mousa Farhadi and Kurosh Sedighi}

Faculty of Mechanical Engineering, Babol Noshirvani University of Technology, Babol, Islamic Republic of Iran

\begin{abstract}
In this paper, biomagnetic fluid flow in a three-dimensional channel in the presence of obstacles and under the influence of a magnetic field is studied numerically. The magnetic field is generated by a wire carrying electric current. The mathematical model of biomagnetic fluid dynamics which is consistent with the principles of ferrohydrodynamics and magnetohydrodynamics is used for the problem formulation. A computational grid which accurately covers the magnetic force is used for the discretisation of computational domain. The flow field is studied in the different arrangements of the obstacles and diverse magnetic field strengths. The results show that the flow pattern is drastically influenced by the applied magnetic field. Applying the magnetic field causes a secondary flow that affects the velocity distribution considerably. The magnetic force also reduces the maximum axial velocity. Furthermore, the magnetic field has a considerable impact on the recirculation zones behind the obstacles. The magnetic field makes the recirculation zones smaller. This study indicates that applying the magnetic field increases the axial drag coefficients of the obstacles significantly (in a case, by $40.15 \%$ ).
\end{abstract}

\section{ARTICLE HISTORY}

Received 12 November 2017 Accepted 19 November 2018

\section{KEYWORDS}

Biomagnetic fluid; computational fluid dynamics; drag coefficient; magnetic field; secondary flow

\section{Introduction}

Biomagnetic fluids are recognised as the biological fluids which magnetic forces have effects on. Biomagnetic fluids' behaviour in the presence of magnetic fields has become an interesting area for many researchers. Cell separation (Haik, Pai, \& Chen, 1999a; Miltenyi, Müller, Weichel, \& Radbruch, 1990), targeted drug delivery (Crane et al., 2004; Cregg, Murphy, \& Mardinoglu, 2012; Lu, Wei, Ma, Yang, \& Chen, 2012; Yellen et al., 2005; Yu, Chen, Chen, \& Zhou, 2008), wound healing due to the effects of magnetic fields (Henry, Concannon, \& Yee, 2008; Nursal et al., 
2006), and cancer treatment with magnetic hyperthermia (Baghban \& Ayani, 2017; Lahiri, Ranoo, \& Philip, 2017; Liangruksa, Ganguly, \& Puri, 2011) are some applications of this field in the medical and biomedical engineering. Blood is the most characteristic biomagnetic fluid. Haemoglobin is a protein-based component of the red blood cells which are primarily responsible for the transportation of oxygen in the human body. Oxygen binds to iron atoms existing in the haemoglobin structure. Studies (Higashi, Ashida, \& Takeuchi, 1997; Higashi et al., 1993; Iino, 1997; Motta, Haik, Gandhari, \& Chen, 1998; Takeuchi, Mizuno, Higashi, Yamagishi, \& Date, 1995) show that the red blood cells orient under the influence of magnetic fields. Binding oxygen to haemoglobin causes change in the magnetic susceptibility of the haemoglobin, so that oxyhaemoglobin behaves as a diamagnetic material, and deoxyhaemoglobin behaves as a paramagnetic material (Shiga, Okazaki, Seiyama, \& Maeda, 1993; Yamagashi, Takeuchi, Hagashi, \& Date, 1992). In general, biological elements have weak magnetic susceptibilities in the order of $10^{-6}$ (Schenck, 1996). To perform some mechanical works on the biological fluids, the magnetic susceptibilities of the fluids could be enhanced, for instance, by labelling the biological cells with magnetic materials (Haik et al., 1999a; Miltenyi et al., 1990).

The study of biomagnetic fluid flow in ducts which are narrowed in some locations affected by magnetic fields is one of the most interesting areas for researchers. Haik, Chen, and Chatterjee (2002) presented biomagnetic fluid's behaviour in a two-dimensional channel with a semi-circular thrombus exposed to a magnetic field. In their study, the centre of the magnetic field is located behind the thrombosis in flow direction. They showed that without the magnetic field a small recirculation zone is formed just behind the thrombus downstream. By applying the magnetic field new recirculation zones are created, and the recirculation zone behind the thrombosis changes. The strength of the recirculation increases as the magnetic field's strength increases. They indicated that when the magnetic field is applied the friction coefficients at the lower and upper walls of the channel increase drastically, and also increase as the magnetic field's strength increases. Khashan and Haik (2006) investigated the magnetic field effects on the biomagnetic fluid downstream an eccentric stenotic orifice two dimensionally. They showed that according to the location of the magnetic field, the reattachment point downstream of the stenotic orifice changes. Tzirtzilakis (2008) studied the biomagnetic fluid (blood) flow in a channel with symmetric stenosis under the action of a magnetic field two dimensionally. He showed the formation of two symmetric vortices downstream of the stenosis in the pure hydrodynamic flows for symmetric stenosis. By applying the magnetic field, the vortex close to the magnetic field source 
is enlarged. Consequently, the reattachment of the flow is shifted downstream. In some cases, one of the vortices appearing in the hydrodynamic case diminishes by applying the magnetic field, and the detachment of the flow does not occur on one of the two plates. Mustapha, Amin, Chakravarty, and Mandal (2009) performed a twodimensional axisymmetric study in terms of the effect of a uniform magnetic field on the blood flow through an artery in the presence of irregularly shaped paired stenoses. Their results show the formation of wall shear stress peaks in the maximum constriction sites. With applying the magnetic field the wall shear stress increases, and with increasing the magnetic force the stress increases. By imposing the magnetic field to the fluid flow, the flow separation can be controlled significantly. Sankar and Lee (2011) analysed the effects of a uniform magnetic field on a pulsatile flow of blood through narrow arteries with mild stenosis. They considered blood as a non-Newtonian fluid. They showed that the skin friction increases significantly in the presence of magnetic field, and the increase of the magnetic force leads to the increase of the skin friction.

Biomagnetic fluid dynamics (BFD) is the biological fluid dynamics in the presence of magnetic fields. Haik, Pai, and Chen (1999b) presented a mathematical model of the BFD based on ferrohydrodynamics (FHD) (Rosensweig, 1985). In this model the magnetic force due to magnetisation, which depends on the gradient of the magnetic field, is considered only. Tzirtzilakis (2005) developed the BFD model by considering the Lorentz force based on the principles of magnetohydrodynamics (MHD) (Shercliff, 1965).

One of the non-uniform magnetic fields is the magnetic field generated by a wire carrying electric current. Mousavi, Farhadi, and Sedighi (2016) presented a method used for finding appropriate computational grids to simulate the effects of this magnetic field on magnetic fluids. They showed that the computational grids must cover the magnetic force accurately.

The aim of the present work is the 3D numerical study of a biomagnetic fluid flow under the influence of a non-uniform magnetic field in a channel in the presence of obstacles. The magnetic field generated by a wire carrying electric current is considered. The obstacles are cubic. In the numerical study of the effects of this magnetic field on the magnetic fluid flow, computational grids play an essential role in the accuracy of obtained results (Mousavi et al., 2016). Therefore, according to the method presented by Mousavi et al. (2016), a computational gird accurately covering the magnetic force is created. This simulation is carried out based on the BFD mathematical model which is consistent with the principles of ferrohydrodynamics 
and magnetohydrodynamics. In this study, the flow fields in the presence of one obstacle, two obstacles in a row, and two obstacles not in a row are analysed. Moreover, axial drag coefficients on the obstacles are specially studied. The analysis of the obtained results indicates that the application of the magnetic field in a magnetic fluid flow could be useful for medical and engineering applications.

\section{Theoretical formulation}

\subsection{Governing equations}

The three-dimensional steady laminar incompressible flow of a homogeneous Newtonian electrical conductor biomagnetic fluid in a channel in the presence of obstacles under the influence of the magnetic field of a wire carrying electric current is considered. A schematic model of the considered problems is shown in Figure 1. The wire with coordinates $(a, b),(\mathrm{a}=0.5 \mathrm{~h}$ and $\mathrm{b}=1.2 \mathrm{~h})$, in the $\mathrm{x}-\mathrm{y}$ plane of the channel is located above the channel parallel to the $\mathrm{z}$ axis (Figure 1).

The viscosity changes due to the applied magnetic field are neglected. Equilibrium magnetisation ( $\mathbf{M}$ and $\mathbf{H}$ parallel) and continuation of magnetic induction i.e. $\nabla \cdot \mathbf{B}=0$ are considered. Given the above assumptions, according to the mathematical model presented by Tzirtzilakis (2005), the equations governing the flow are as follows:

$$
\begin{aligned}
& \frac{\partial u}{\partial x}+\frac{\partial v}{\partial y}+\frac{\partial w}{\partial z}=0 \\
& \rho\left(u \frac{\partial u}{\partial x}+v \frac{\partial u}{\partial y}+w \frac{\partial u}{\partial z}\right)=-\frac{\partial p}{\partial x}+\mu\left(\frac{\partial^{2} u}{\partial x^{2}}+\frac{\partial^{2} u}{\partial y^{2}}+\frac{\partial^{2} u}{\partial z^{2}}\right)+\mu_{0} M \frac{\partial H}{\partial x} \\
&+\sigma\left(v B_{x} B_{y}-u B_{y}^{2}\right) \\
& \rho\left(u \frac{\partial v}{\partial x}+v \frac{\partial v}{\partial y}+w \frac{\partial v}{\partial z}\right)=-\frac{\partial p}{\partial y}+\mu\left(\frac{\partial^{2} v}{\partial x^{2}}+\frac{\partial^{2} v}{\partial y^{2}}+\frac{\partial^{2} v}{\partial z^{2}}\right)+\mu_{0} M \frac{\partial H}{\partial y} \\
&+\sigma\left(u B_{x} B_{y}-v B_{x}^{2}\right) \\
& \rho\left(u \frac{\partial w}{\partial x}+v \frac{\partial w}{\partial y}+w \frac{\partial w}{\partial z}\right)=-\frac{\partial p}{\partial z}+\mu\left(\frac{\partial^{2} w}{\partial x^{2}}+\frac{\partial^{2} w}{\partial y^{2}}+\frac{\partial^{2} w}{\partial z^{2}}\right)-\sigma\left(w B^{2}\right)
\end{aligned}
$$

A laminar fully developed flow with average axial velocity $\bar{W}_{0}$ enters the channel (at $\mathrm{z}=0$ ), and diffusion fluxes for all flow variables normal to the exit plane are assumed to be zero at the outlet of the channel $(z=L)$. The no-slip condition is imposed on the walls. 

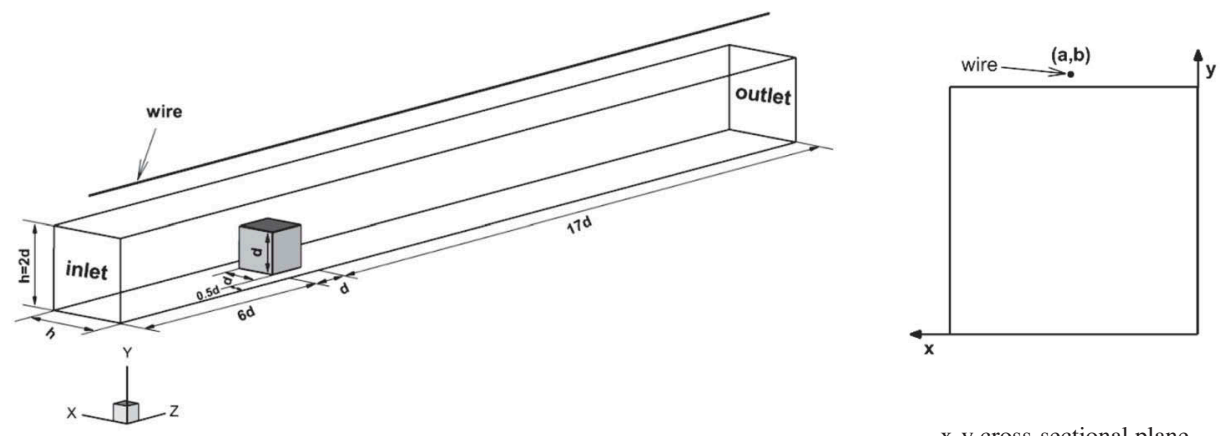

$\mathrm{x}$-y cross-sectional plane

(a)

(b)
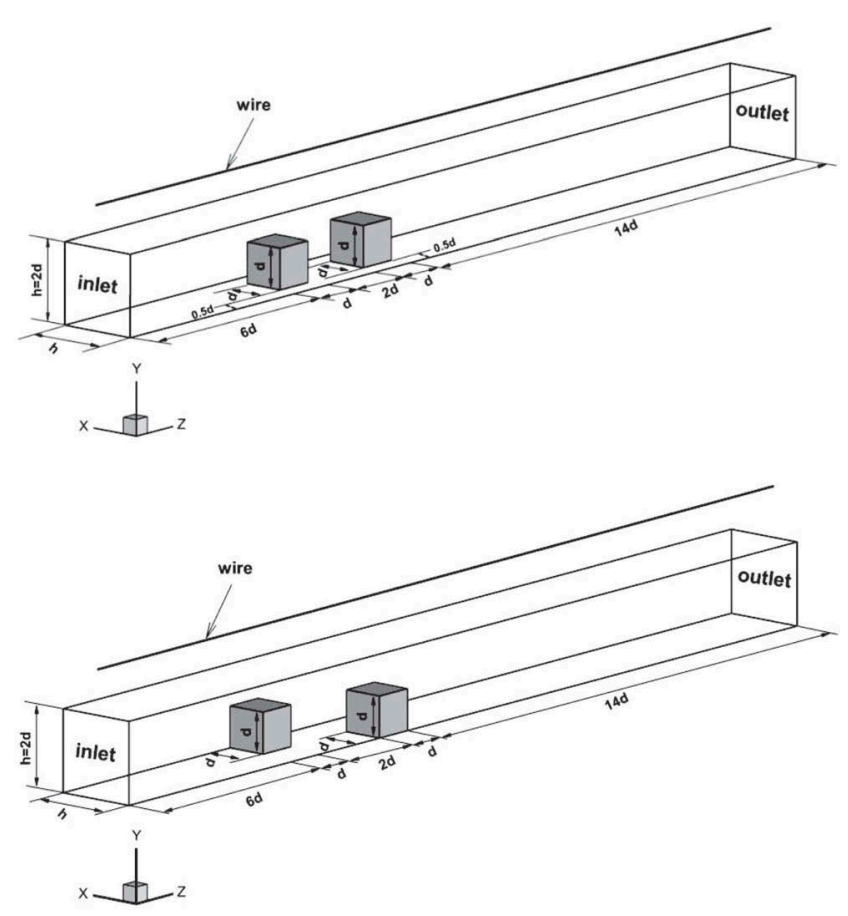

Figure 1. Schematic models of the considered problems. Channel with (a) one obstacle, (b) two obstacles in a row, (c) two obstacles not in a row.

In the above equations, $\mathbf{V}=(u, v, w)$ is the velocity field, $\rho$ is the biomagnetic fluid density, $p$ is the pressure, $\mu$ is the biomagnetic fluid dynamic viscosity, $\mu_{0}$ is the magnetic permeability of vacuum, $\mathbf{M}$ is the magnetisation, $\mathbf{H}$ is the magnetic field intensity, $\mathbf{B}$ is the magnetic field induction $\left(B_{x}\right.$ and $B_{y}$ are the magnetic field induction components along the $\mathrm{x}$ and $\mathrm{y}$ directions, respectively) and $\sigma$ is the biomagnetic fluid electrical conductivity.

The terms $\mu_{0} M \partial H / \partial x+\sigma\left(v B_{x} B_{y}-u B_{y}^{2}\right), \quad \mu_{0} M \partial H / \partial y+\sigma\left(u B_{x} B_{y}-\right.$ $\left.v B_{x}^{2}\right)$, and $-\sigma\left(w B^{2}\right)$ in Equations (2)-(4) represent the components of 
the magnetic force per unit volume along the $\mathrm{x}, \mathrm{y}$, and $\mathrm{z}$ directions, respectively. $\mu_{0} M \partial H / \partial x$ and $\mu_{0} M \partial H / \partial y$ are due to the magnetisation that depends on the existence of the magnetic gradient. $\sigma\left(v B_{x} B_{y}-u B_{y}{ }^{2}\right)$, $\sigma\left(u B_{x} B_{y}-v B_{x}^{2}\right)$, and $-\sigma\left(w B^{2}\right)$ represent the components of the Lorentz force per unit volume (the Lorentz force per unit volume is equal to $\mathbf{J} \times \mathbf{B}$ where $\mathbf{J}$ is the density of the electric current, and $\nabla \times \mathbf{H}=\mathbf{J}=\sigma(\mathbf{V} \times \mathbf{B}))$ which are due to the induced electric current.

The relation between the magnetic field induction and magnetic field intensity is as follows:

$$
\mathbf{B}=\mu_{0} \mathbf{H}
$$

The magnetisation as a linear equation (Tzirtzilakis, 2005) is considered as follows:

$$
\mathbf{M}=\chi \mathbf{H}
$$

where $\chi$ is the magnetic susceptibility of the biomagnetic fluid.

The magnetic field intensity components $H_{x}$ and $H_{y}$ along the $\mathrm{x}$ and y directions are as follows:

$$
\begin{aligned}
H_{x} & =\frac{I}{2 \pi} \frac{y-b}{(\mathrm{x}-a)^{2}+(y-b)^{2}} \\
H_{y} & =\frac{I}{2 \pi} \frac{x-a}{(\mathrm{x}-a)^{2}+(y-b)^{2}}
\end{aligned}
$$

where $(a, b)$ is the position of the wire in the $\mathrm{x}$-y plane, and $I$ is the electric current intensity.

The magnitude of the magnetic field intensity is given by

$$
H=\frac{I}{2 \pi} \frac{1}{\sqrt{(x-a)^{2}+(y-b)^{2}}}
$$

The dimensionless variables are defined as follows:

$$
\begin{aligned}
& x^{*}=\frac{x}{h}, y^{*}=\frac{y}{h}, z^{*}=\frac{z}{h}, u^{*}=\frac{u}{W_{0}}, v^{*}=\frac{v}{W_{0}}, w^{*}=\frac{w}{W_{0}} \\
& , p^{*}=\frac{p}{\rho \bar{W}_{0}{ }^{2}}, H_{x}{ }^{*}=\frac{H_{x}}{H_{0}}, H_{y}{ }^{*}=\frac{H_{y}}{H_{0}}
\end{aligned}
$$

The dimensionless forms of equations are

$$
\begin{gathered}
\frac{\partial u^{*}}{\partial x^{*}}+\frac{\partial v^{*}}{\partial y^{*}}+\frac{\partial w^{*}}{\partial z^{*}}=0 \\
u^{*} \frac{\partial u^{*}}{\partial x^{*}}+v^{*} \frac{\partial u^{*}}{\partial y^{*}}+w^{*} \frac{\partial u^{*}}{\partial z^{*}}=-\frac{\partial p^{*}}{\partial x^{*}}+\frac{1}{\operatorname{Re}}\left(\frac{\partial^{2} u^{*}}{\partial x^{* 2}}+\frac{\partial^{2} u^{*}}{\partial y^{* 2}}+\frac{\partial^{2} u^{*}}{\partial z^{* 2}}\right)+\operatorname{Mn} H^{*} \frac{\partial H^{*}}{\partial x^{*}} \\
+\mathrm{N}\left(v^{*} H_{x}{ }^{*} H_{y}{ }^{*}-u^{*} H_{y}{ }^{* 2}\right)
\end{gathered}
$$




$$
\begin{gathered}
u^{*} \frac{\partial v^{*}}{\partial x^{*}}+v^{*} \frac{\partial v^{*}}{\partial y^{*}}+w^{*} \frac{\partial v^{*}}{\partial z^{*}}=-\frac{\partial p^{*}}{\partial y^{*}}+\frac{1}{\operatorname{Re}}\left(\frac{\partial^{2} v^{*}}{\partial x^{* 2}}+\frac{\partial^{2} v^{*}}{\partial y^{* 2}}+\frac{\partial^{2} v^{*}}{\partial z^{* 2}}\right)+\mathrm{Mn} H^{*} \frac{\partial H^{*}}{\partial y^{*}} \\
+\mathrm{N}\left(u^{*} H_{x}{ }^{*} H_{y}{ }^{*}-v^{*} H_{x}{ }^{2}\right) \\
\begin{aligned}
u^{*} \frac{\partial w^{*}}{\partial x^{*}}+v^{*} \frac{\partial w^{*}}{\partial y^{*}}+w^{*} \frac{\partial w^{*}}{\partial z^{*}}= & -\frac{\partial p^{*}}{\partial z^{*}}+\frac{1}{\operatorname{Re}}\left(\frac{\partial^{2} w^{*}}{\partial x^{* 2}}+\frac{\partial^{2} w^{*}}{\partial y^{* 2}}+\frac{\partial^{2} w^{*}}{\partial z^{* 2}}\right) \\
& -\mathrm{N}\left(w^{*} H^{* 2}\right)
\end{aligned}
\end{gathered}
$$

The dimensionless numbers are

$$
\operatorname{Re}=\frac{\rho \bar{W}_{0} h}{\mu} \mathrm{Mn}=\frac{\mu_{0} \chi H_{0}^{2}}{\rho \bar{W}_{0}^{2}}=\frac{\chi B_{0}^{2}}{\mu_{0} \rho \bar{W}_{0}^{2}} \mathrm{~N}=\frac{\mu_{0}{ }^{2} H_{0}{ }^{2} h \sigma}{\rho \bar{W}_{0}}=\frac{B_{0}{ }^{2} h \sigma}{\rho \bar{W}_{0}}=\frac{\mathrm{Ha}^{2}}{\operatorname{Re}}
$$

where $\mathrm{Re}$ is the Reynolds number, $\mathrm{Mn}$ is the magnetic number, and $\mathrm{N}$ is the Stuart number which is the ratio of the square of the Hartmann number $(\mathrm{Ha})$ to the Reynolds number. $H_{0}$ and $B_{0}$ are the magnetic field intensity and magnetic field induction at the point $(a, h)$, respectively, and $B_{0}=\mu_{0} H_{0}$.

\subsection{Numerical method}

In the present study, the computational fluid dynamics software ANSYS FLUENT was employed to solve the mentioned equations in the previous section using the finite volume method. To apply the magnetic forces some User-Defined Functions (UDFs) were written and added to the software. The SIMPLE algorithm was used for the velocity-pressure coupling. The convective and diffusive terms in the momentum equations were discretised by using the second order upwind method. A structured nonuniform grid was used for the discretisation of computational domain. To ensure that the solution is not affected by the computational grid size, grid independency is analysed. According to the Mousavi, et al.'s work (2016) the computational grids covering the magnetic force relatively accurately were created. The grid independency in the $x, y$, and $\mathrm{z}$ directions in the presence of two obstacles in a row (Figure 1(b)) was investigated by choosing the dimensionless axial velocity, and the results are shown in Figure 2. According to the results, the non-uniform grid $175 \times 125 \times 130$ was selected as a suitable grid. As can be seen in Figure 2, with increasing the number of grid points, the dimensionless axial velocity does not change significantly. 


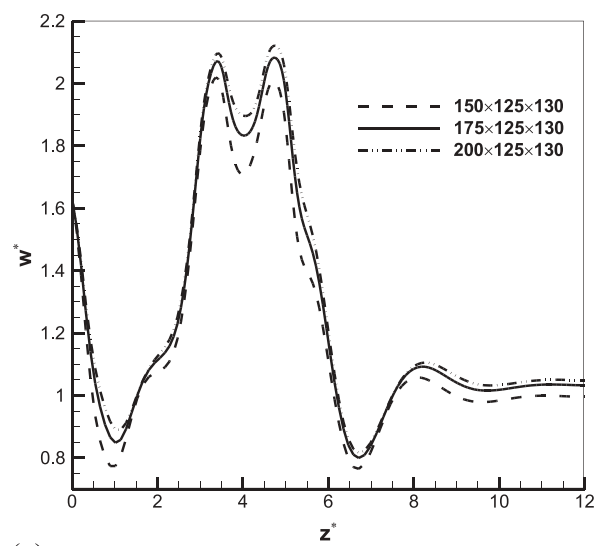

(a)

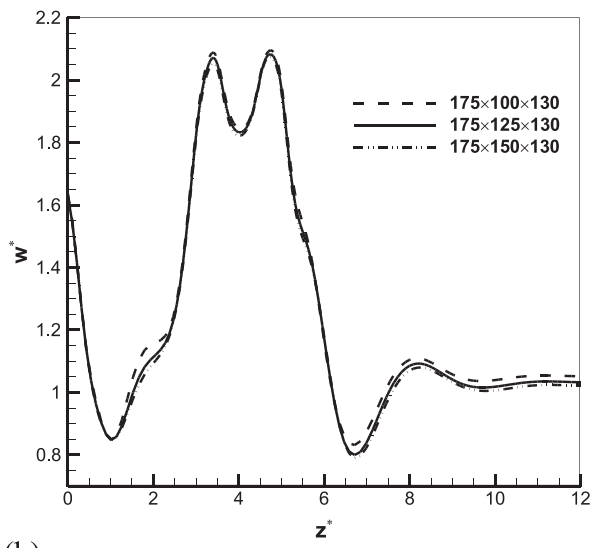

(b)

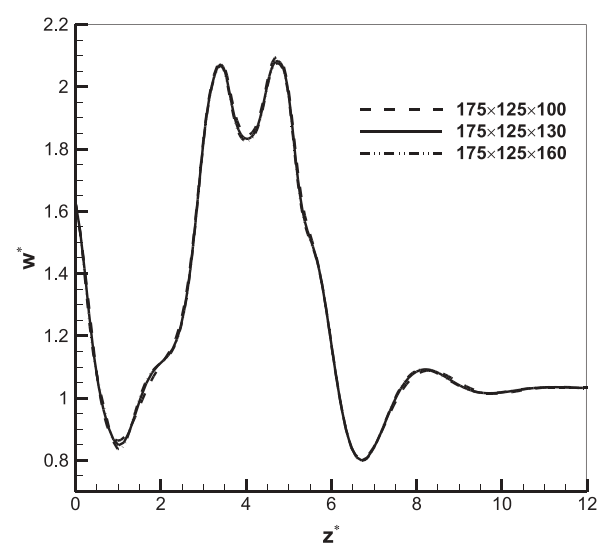

(c)

Figure 2. The comparison of the dimensionless axial velocity for $\operatorname{Re}=50, \mathrm{Mn}=1.0 \times 10^{5}$, and $\mathrm{N}=6.065 \times 10^{-2}$ on the axial line at $x^{*}=.5$ and $y^{*}=0.75$ in the different grid points (the grids are non-uniform) along the (a) $x^{*}$ direction, (b) $y^{*}$ direction, (c) $z^{*}$ direction.

\section{Results and discussion}

\subsection{One obstacle}

In the presence of one obstacle, the streamlines are shown in Figure 3. In the absence of the magnetic field, the streamlines are mainly parallel to the z-axis (Figure 3(a)). By applying the magnetic field a secondary flow is created. As shown in Figure 3(b), the streamlines are not just parallel to the $\mathrm{z}$-axis, and the streamlines in the form of recirculation zones are visible in the $x-y$ cross sectional planes of the channel. Figure 4 shows the streamlines in the $y-z$ cross sectional plane of the channel $\left(\right.$ at $\left.x^{*}=.5\right)$ in the various magnetic field strengths. The presence of obstacle changes the pressure distribution. Positive pressure gradient occurs downstream of the obstacle, which causes the creation of a recirculation zone. By applying the magnetic field, the streamlines are driven towards the bottom wall 

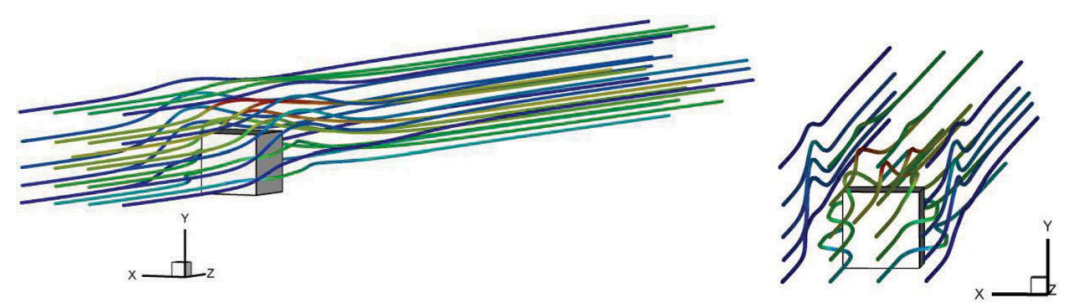

(a) $\mathrm{Mn}=0$ and $\mathrm{N}=0$
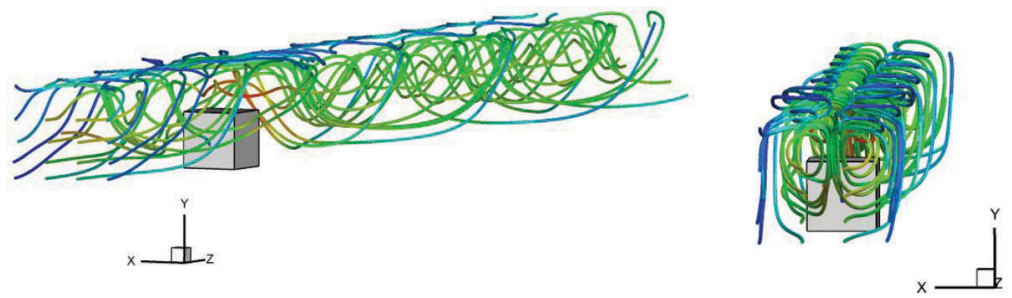

(b) $\mathrm{Mn}=6.4 \times 10^{4}$ and $\mathrm{N}=3.881 \times 10^{-2}$

Figure 3. Streamlines at $\mathrm{Re}=50$.

of the channel. This flow movement towards the bottom wall of the channel affects the pressure gradient and can change the recirculation zone. With increasing the magnetic force the vortex located downstream of the obstacle becomes smaller. Since the magnetic field is applied throughout the channel, the flow from upstream to downstream of the obstacle is changed. When the flow in upstream of the obstacle, which comes down, reaches the obstacle is forced to pass through the sides of the obstacle as shown in Figure 5. The flow in the region close to the magnetic source is driven downwards sharply. As can be seen in Figure 5, by applying the magnetic field the flow hits the middle of channel's bottom wall $\left(\mathrm{x}^{*}=.5\right)$. In Figure 6 the streamlines are shown in the two perpendicular longitudinal sections. In Figure 6(b) the flow driven to the bottom of the channel by the magnetic force and the flow passed through the side of the obstacle are obvious. Figure 7 shows the streamlines in the transverse sections before and on the obstacle. As can be seen in the Figure 7, the magnetic field influences the flow pattern, and a flow in the direction of the magnetic force is created which causes the secondary flow. The flow towards the bottom of the channel causes the recirculation zones in the upper corners of the channel. The axial velocity distribution in the transverse sections before and on the obstacle in the absence and presence of the magnetic field is shown in Figure 8. It can be seen that applying the magnetic field has a significant impact on the velocity distribution. The comparison of the axial velocity distributions in the absence and presence of the magnetic field indicates that in the absence 


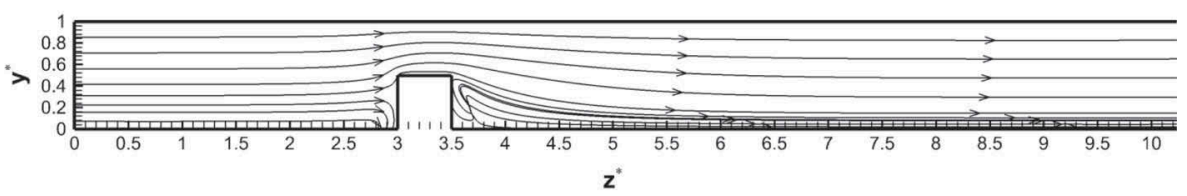

(a) $\mathrm{Mn}=0$ and $\mathrm{N}=0$

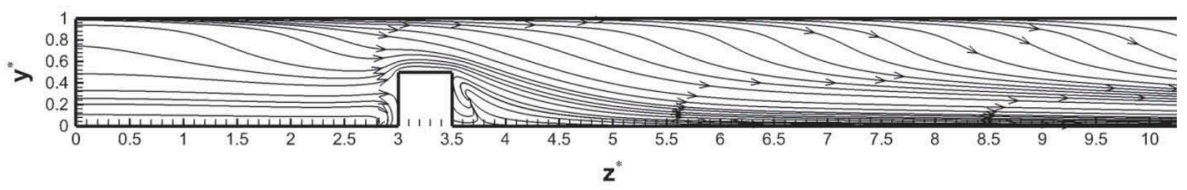

(b) $\mathrm{Mn}=1.6 \times 10^{4}$ and $\mathrm{N}=9.703 \times 10^{-3}$

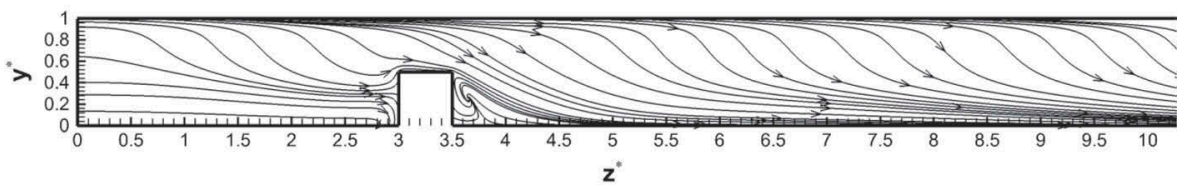

(c) $\mathrm{Mn}=3.6 \times 10^{4}$ and $\mathrm{N}=2.183 \times 10^{-2}$

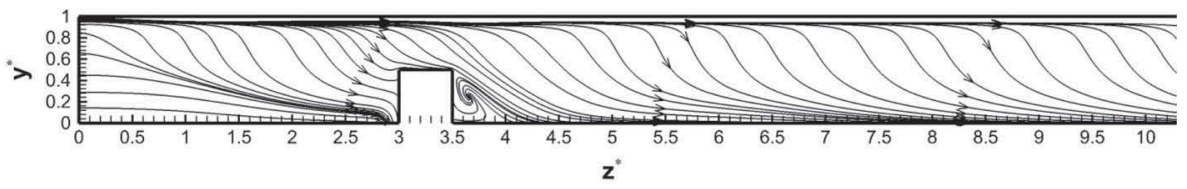

(d) $\mathrm{Mn}=6.4 \times 10^{4}$ and $\mathrm{N}=3.881 \times 10^{-2}$

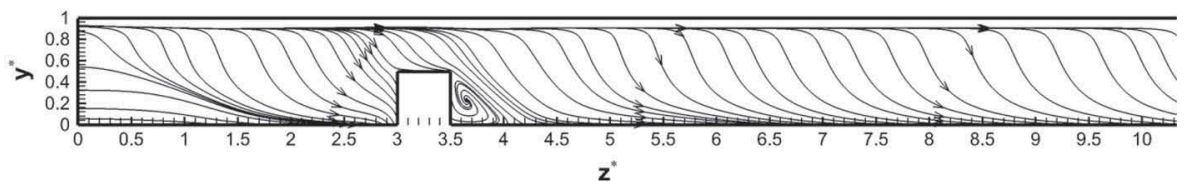

(e) $\mathrm{Mn}=1.0 \times 10^{5}$ and $\mathrm{N}=6.065 \times 10^{-2}$

Figure 4. Streamlines in the $y-z$ cross sectional plane at $x^{*}=0.5$ and $\operatorname{Re}=50$.

of the magnetic field the maximum velocity is higher. In the presence of the magnetic field, the flow goes towards the bottom of the channel, and this causes maximum axial velocity reduction.

The drag coefficient $C_{D}$ is calculated by the following relation:

$$
C_{D}=\frac{F_{D}}{\frac{1}{2} \rho \bar{W}_{0}^{2} A}
$$




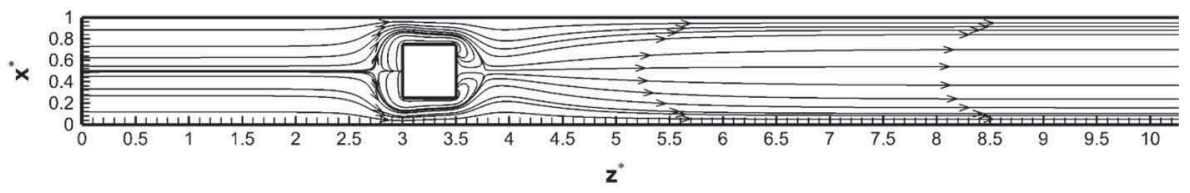

(a) $\mathrm{Mn}=0$ and $\mathrm{N}=0$

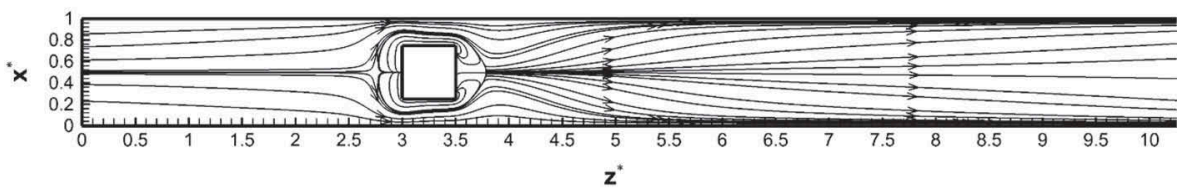

(b) $\mathrm{Mn}=1.6 \times 10^{4}$ and $\mathrm{N}=9.703 \times 10^{-3}$

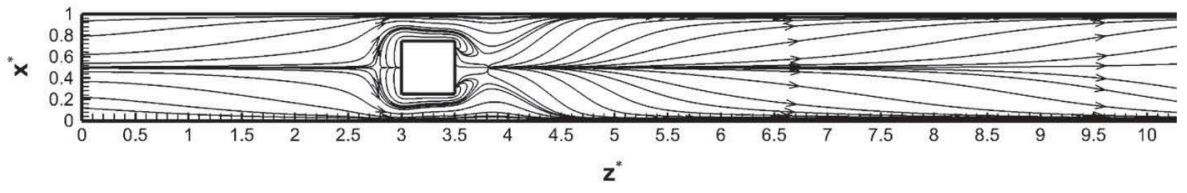

(c) $\mathrm{Mn}=3.6 \times 10^{4}$ and $\mathrm{N}=2.183 \times 10^{-2}$

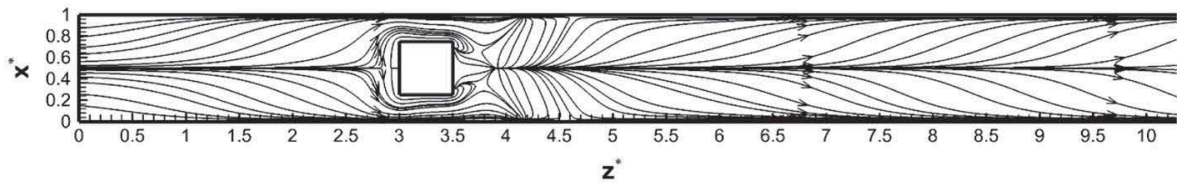

(d) $\mathrm{Mn}=6.4 \times 10^{4}$ and $\mathrm{N}=3.881 \times 10^{-2}$

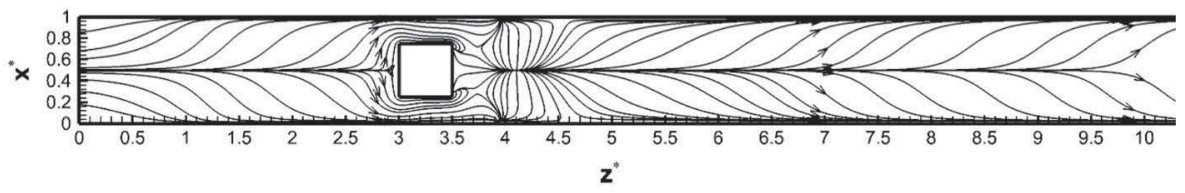

(e) $\mathrm{Mn}=1.0 \times 10^{5}$ and $\mathrm{N}=6.065 \times 10^{-2}$

Figure 5. Streamlines on the bottom of the channel at $\operatorname{Re}=50$.

where $F_{D}$ is the drag force, and $A$ is the surface area.

In Figure 9, the influence of applying the magnetic field on the obstacle drag coefficient in the axial direction is shown. In this figure the values of $C_{D} / C_{D 0}$ are shown at different values of $\mathrm{Mn}$, where $C_{D 0}$ is the obstacle drag coefficient in the absence of the magnetic field. With increasing the magnetic force the obstacle drag coefficient increases so that at $\mathrm{Mn}=1.0 \times 10^{5}$ the obstacle drag coefficient increases by $22.14 \%$ compared to the obstacle drag coefficient in the absence of the magnetic 


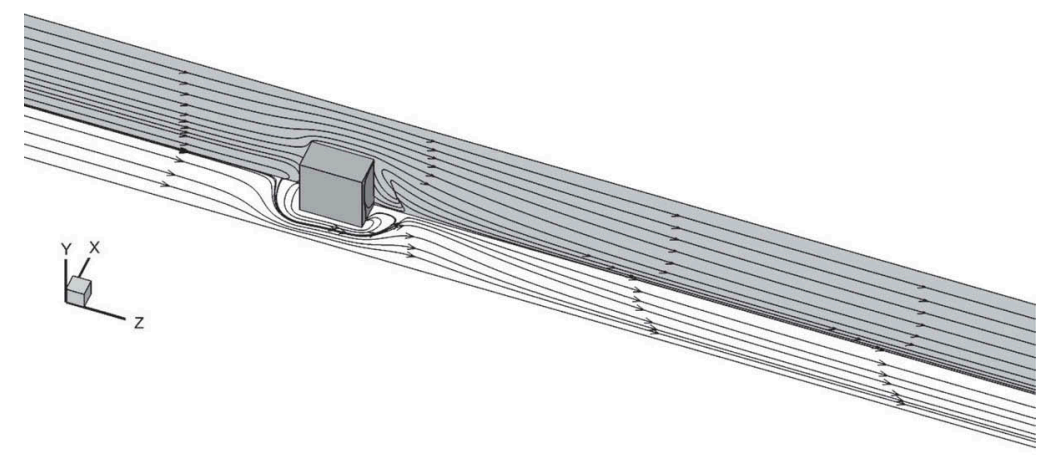

(a) $\mathrm{Mn}=0$ and $\mathrm{N}=0$

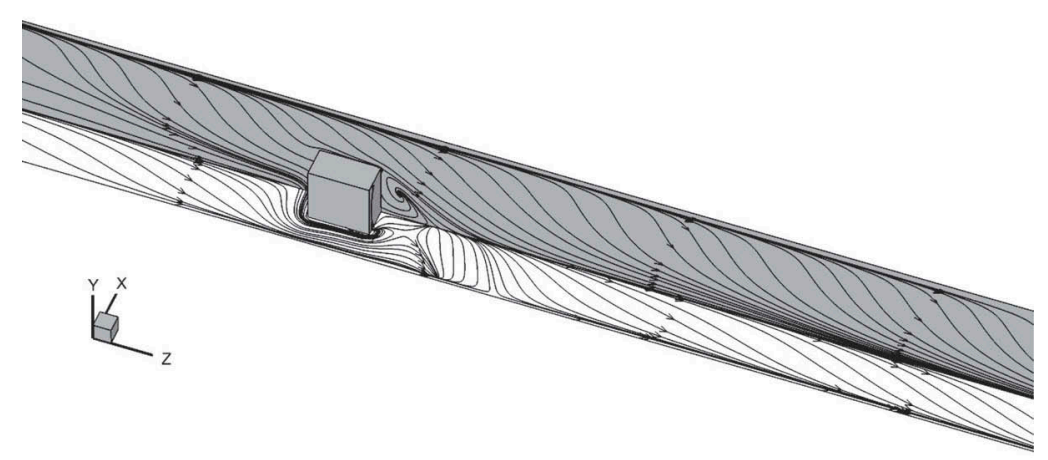

(b) $\mathrm{Mn}=6.4 \times 10^{4}$ and $\mathrm{N}=3.881 \times 10^{-2}$

Figure 6. Streamlines on longitudinal sections at $x^{*}=.5$ and $y^{*}=$ for $\operatorname{Re}=50$.

field. The total drag comprises skin friction drag and pressure drag. Applying the magnetic field causes a downward flow leading to the reduction of the recirculation zone behind the obstacle. This phenomenon reduces the pressure drag, but applying the magnetic field increases shear stress significantly as Mousavi et al. (2016) reported. Therefore, the skin friction drag increases significantly. According to our results, in $\operatorname{Re}=50$, the increase of frictional drag outweighs the decrease of pressure drag.

\subsection{Two obstacles in a row}

Figure 10 depicts the streamlines in the presence of two obstacles in a row, in the $y-z$ cross sectional plane of the channel $\left(\right.$ at $\left.x^{*}=.5\right)$ in the absence and presence of the magnetic field. In the absence of the magnetic field a vortex is formed downstream of each obstacle (Figure 10(a)). Applying the magnetic field drives the flow towards the bottom of the channel. This movement affects the vortexes and makes them smaller (Figure 10). In Figure 11, the effect of the 


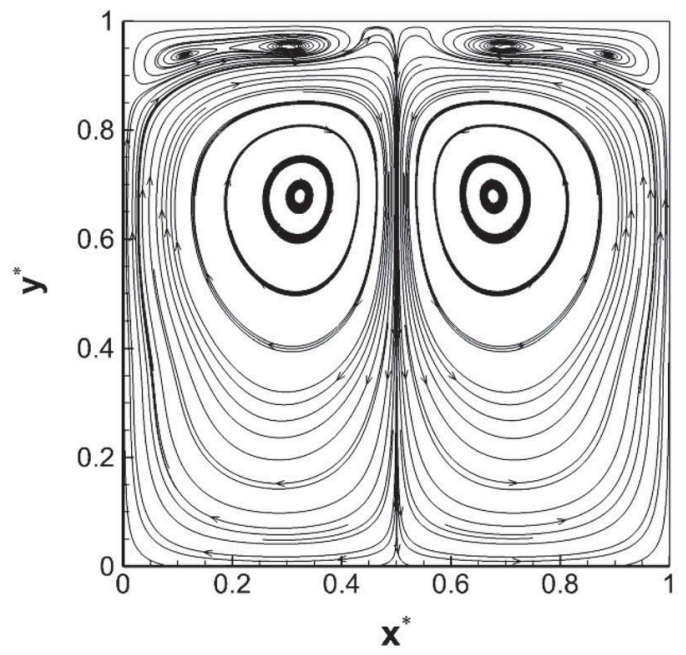

(a) $z^{*}=2$

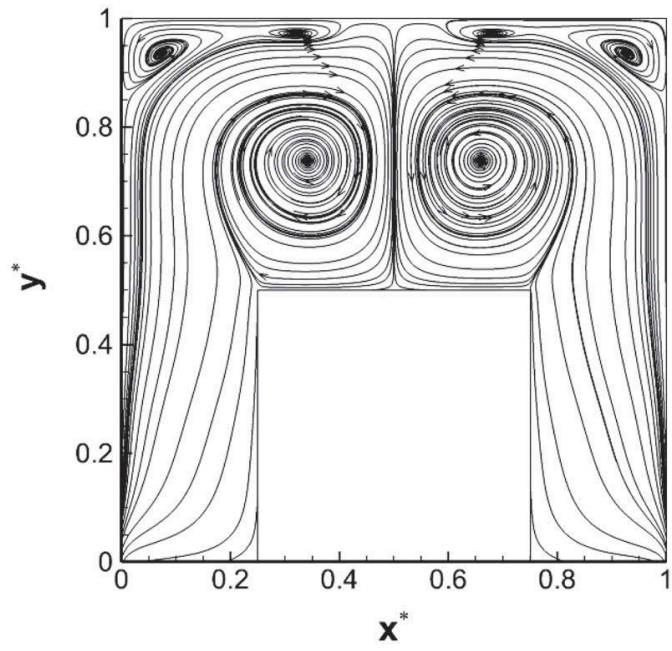

(a) $\mathrm{z}^{*}=3.25$

Figure 7. Streamlines on transverse sections at $\operatorname{Re}=50, \mathrm{Mn}=3.6 \times 10^{4}$, and $\mathrm{N}=2.183 \times 10^{-2}$.

magnetic field on the flow in the bottom of the channel is shown. The downward flow and the flow passing through the sides of the obstacles can be seen in Figure 12. In Figure 13, the axial velocity distributions in the transverse section between the two obstacles in the absence and presence of the magnetic field are shown. It can clearly be seen that by applying the magnetic field, the maximum of the axial velocity is decreased and the velocity distribution is changed enormously.

The calculation of the drag coefficients in the axial direction shows that at $\mathrm{Mn}=6.4 \times 10^{4}$ the drag coefficient of the first obstacle increases 

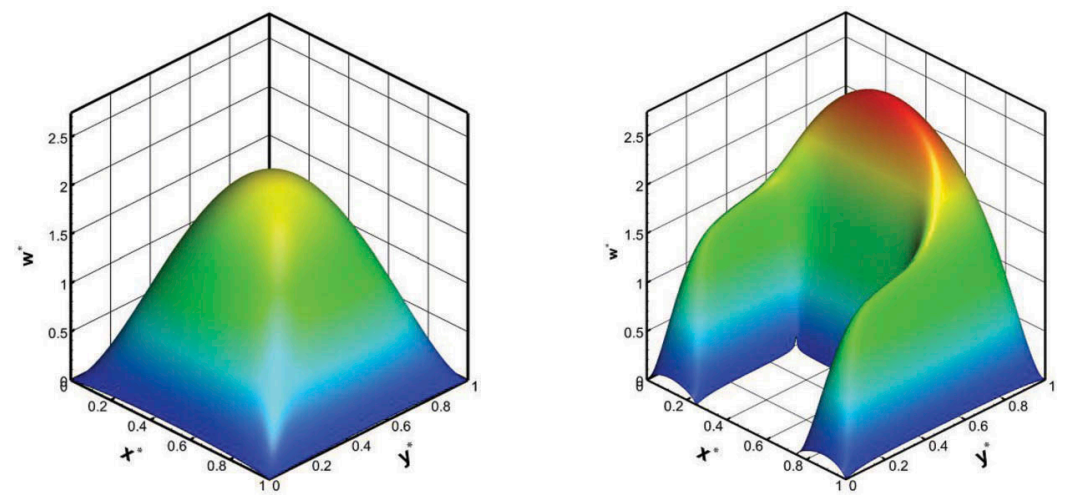

$\mathrm{z}^{*}=2$

$\mathrm{z}^{*}=3.25$

(a) $\mathrm{Mn}=0$ and $\mathrm{N}=0$
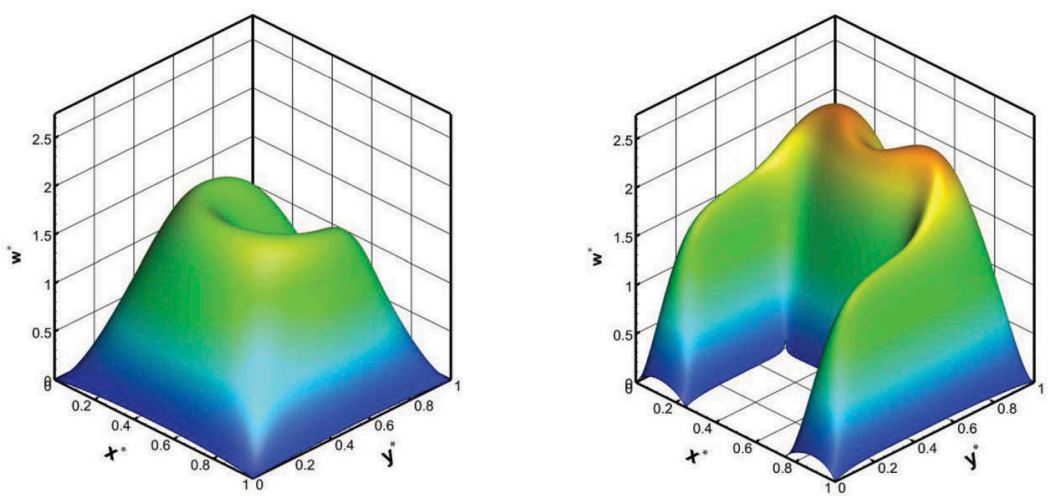

$\mathrm{z}^{*}=2$

$\mathrm{z}^{*}=3.25$

(b) $\mathrm{Mn}=3.6 \times 10^{4}$ and $\mathrm{N}=2.183 \times 10^{-2}$

Figure 8. Dimensionless axial velocity at $\mathrm{Re}=50$.

by $14.22 \%$ compared to the drag coefficient of this obstacle in the absence of the magnetic field. Moreover, the drag coefficient of the second obstacle increases by $40.15 \%$ compared to the drag coefficient of this obstacle in the absence of the magnetic field. The increase in second obstacle's drag coefficient is extremely significant. According to Figure 10, in the absence of the magnetic field, the area in front of the second obstacle is in the wake region of the first obstacle. Applying the magnetic field causes a downward flow leading to the decrease of the wake region and increase of the pressure in front of the second obstacle. Therefore, in this case, applying the magnetic field increases not only frictional drag but also pressure drag, which causes a dramatic rise in the total drag on the second obstacle. 


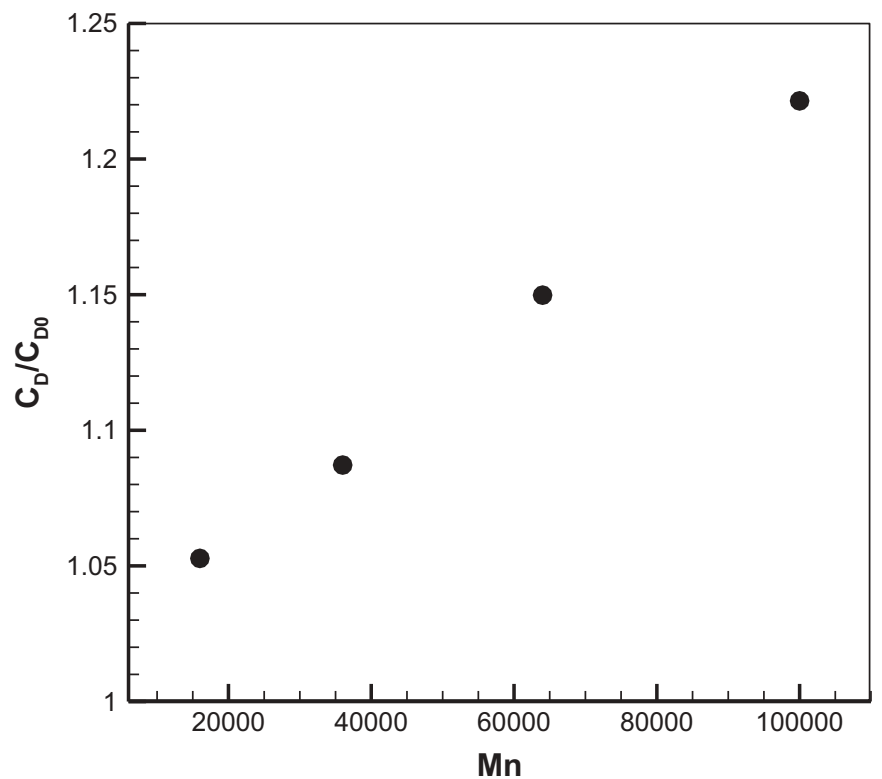

Figure 9. Relative drag coefficient of the obstacle in the different strengths of the magnetic field at $\operatorname{Re}=50$.

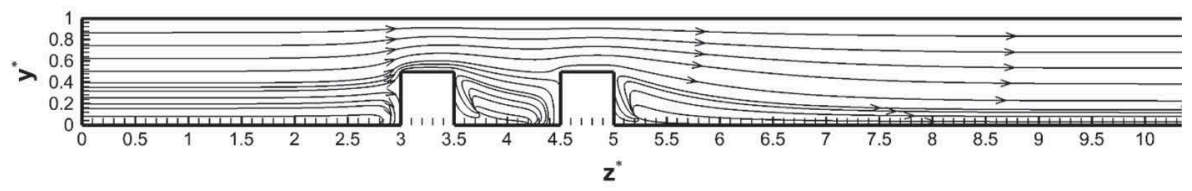

(a) $\mathrm{Mn}=0$ and $\mathrm{N}=0$

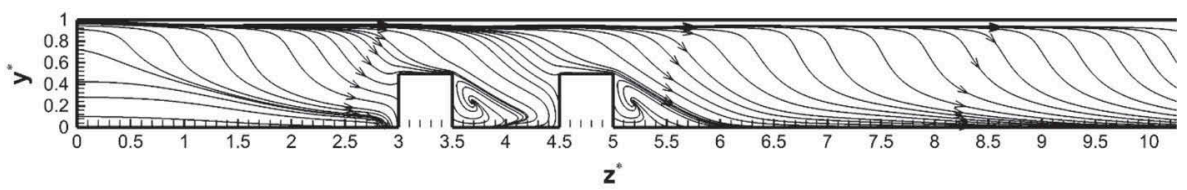

(b) $\mathrm{Mn}=6.4 \times 10^{4}$ and $\mathrm{N}=3.881 \times 10^{-2}$

Figure 10. Streamlines on the $y-z$ cross sectional plane at $x^{*}=.5$ and $\operatorname{Re}=50$.

\subsection{Two obstacles not in a row}

Figure 14 depicts the streamlines in the presence of two obstacles not in a row, in the side and bottom walls of the channel. In Figure 14(b), the confluence of the flows passing through the side of the obstacle, and moving towards the bottom of the channel is quite apparent. To show the influence of the magnetic field on the flow pattern between two obstacles, the axial velocity distributions in the transverse section between the two obstacles in the absence and presence of the magnetic field are shown in Figure 15. 


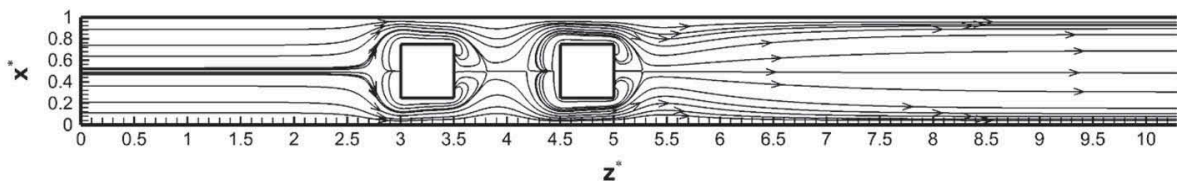

(a) $\mathrm{Mn}=0$ and $\mathrm{N}=0$

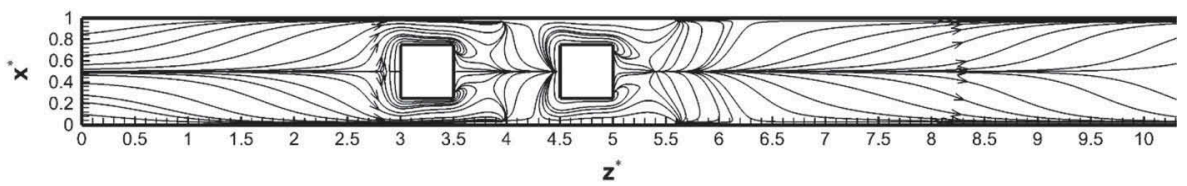

(b) $\mathrm{Mn}=6.4 \times 10^{4}$ and $\mathrm{N}=3.881 \times 10^{-2}$

Figure 11. Streamlines on the bottom of the channel at $\operatorname{Re}=50$.

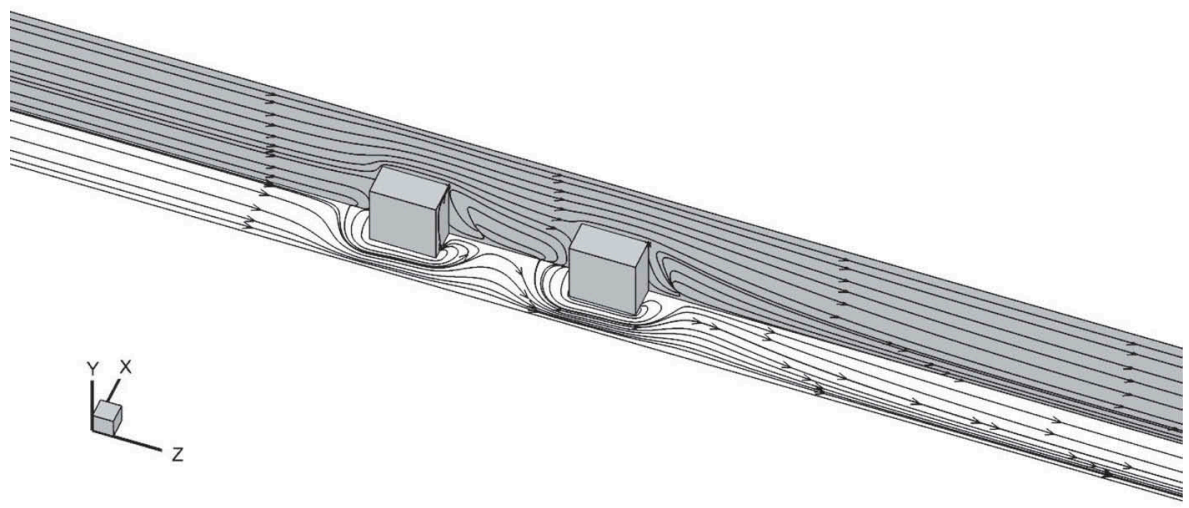

(a) $\mathrm{Mn}=0$ and $\mathrm{N}=0$

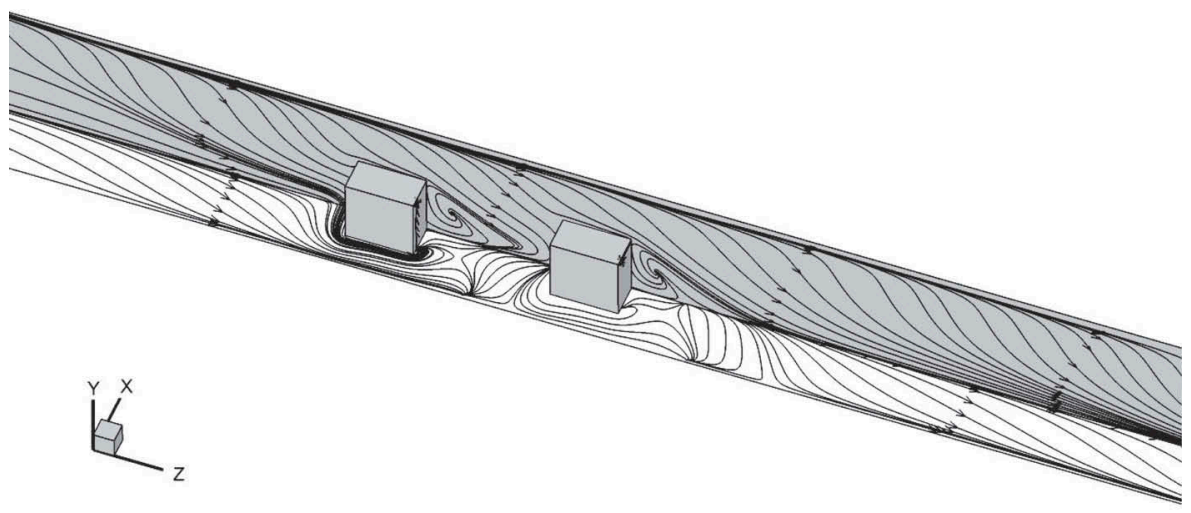

(b) $\mathrm{Mn}=6.4 \times 10^{4}$ and $\mathrm{N}=3.881 \times 10^{-2}$

Figure 12. Streamlines on longitudinal sections at $x^{*}=0.5$ and $y^{*}=0$ in $\operatorname{Re}=50$. 


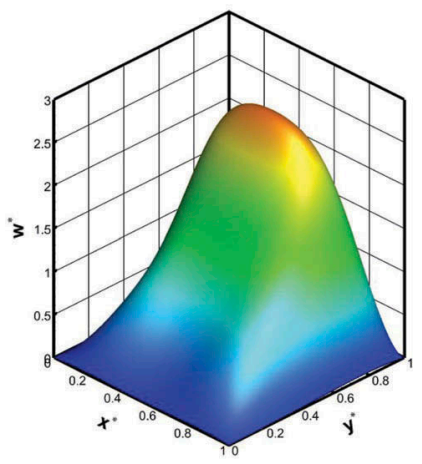

(a) $\mathrm{Mn}=0$ and $\mathrm{N}=0$

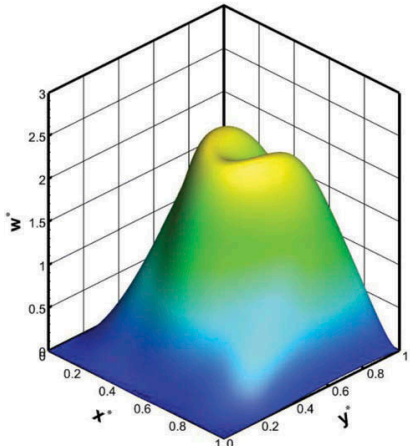

(b) $\mathrm{Mn}=3.6 \times 10^{4}$ and $\mathrm{N}=2.183 \times 10^{-2}$

Figure 13. Dimensionless axial velocity at $z^{*}=4$ and $\operatorname{Re}=50$.

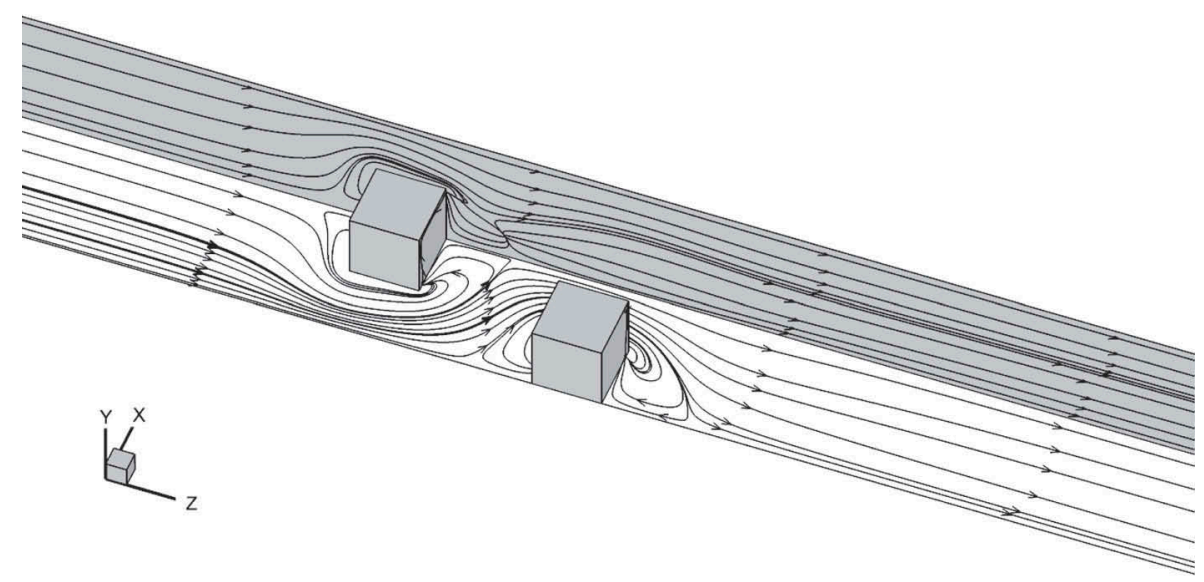

(a) $\mathrm{Mn}=0$ and $\mathrm{N}=0$

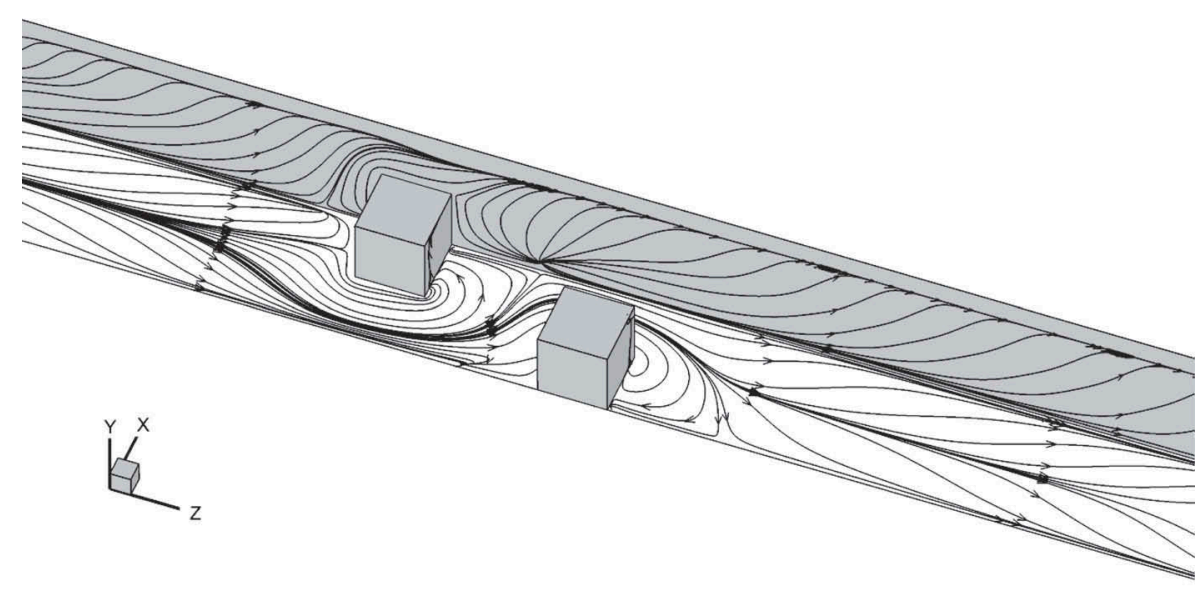

(b) $\mathrm{Mn}=6.4 \times 10^{4}$ and $\mathrm{N}=3.881 \times 10^{-2}$

Figure 14. Streamline on longitudinal sections at $x^{*}=1$ and $y^{*}=0$ in $\operatorname{Re}=50$. 


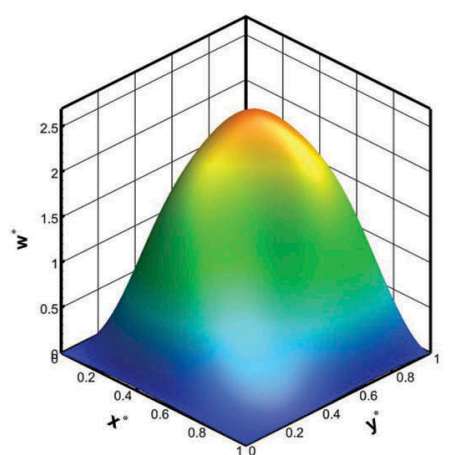

(a) $\mathrm{Mn}=0$ and $\mathrm{N}=0$

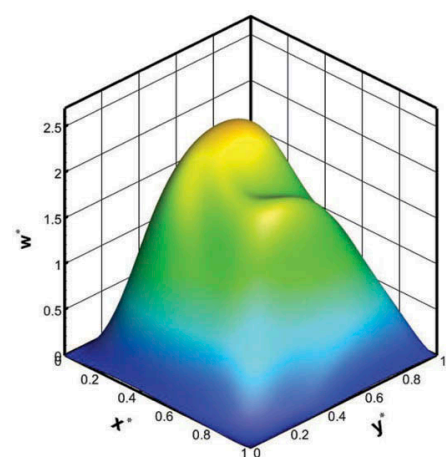

(b) $\mathrm{Mn}=3.6 \times 10^{4}$ and $\mathrm{N}=2.183 \times 10^{-2}$

Figure 15. Dimensionless axial velocity at $z^{*}=4$ and $\mathrm{Re}=50$.

The calculation of the drag coefficients in the axial direction shows that at $\mathrm{Mn}=6.4 \times 10^{4}$ the drag coefficient of the first obstacle increases by $19.42 \%$ compared to the drag coefficient of this obstacle in the absence of the magnetic field. Furthermore, the drag coefficient of the second obstacle increases by $24.42 \%$ compared to the drag coefficient of this obstacle in the absence of the magnetic field.

\section{Conclusion}

In this study, the effect of the magnetic field generated by a wire carrying electric current on the biomagnetic fluid flow is studied numerically. The flow in a three-dimensional channel in the presence of one obstacle, two obstacles in a row, and two obstacles not in a row is investigated. The wire carrying electric current is located above the channel. The following conclusions are obtained from the results:

- Applying the magnetic field causes a secondary flow, and therefore velocity distribution is changed.

- The magnetic field completely affects the flow, and considerably changes the recirculation zones downstream of the obstacles. The flow is driven towards the bottom wall of the channel by the magnetic force, which makes the recirculation zones downstream of the obstacles smaller.

- Evaluating the drag coefficient in the axial direction in the presence of an obstacle in the channel indicates that with increasing the magnetic force the drag coefficient also increases. The drag coefficient at $\mathrm{Mn}=6.4 \times 10^{4}$ increases by $14.98 \%$ and at $\mathrm{Mn}=1.0 \times 10^{5}$ increases by $22.14 \%$ compared to the drag coefficient in the absence of the magnetic field. 
- In the presence of the two obstacles in a row in the axial direction and at $\mathrm{Mn}=6.4 \times 10^{4}$ the drag coefficient of the first obstacle increases by $14.22 \%$, and the drag coefficient of the second obstacle increases by $40.15 \%$ compared to the drag coefficient of the same obstacles in the absence of the magnetic field. The increase of second obstacle's drag coefficient in the presence of the magnetic field is noticeable.

- In the presence of the two obstacles not in a row in the axial direction and at $\mathrm{Mn}=6.4 \times 10^{4}$ the drag coefficient of the first obstacle increases by $19.42 \%$, and the drag coefficient of the second obstacle increases by $24.42 \%$ compared to the drag coefficient of the same obstacles in the absence of the magnetic field.

\section{Disclosure statement}

No potential conflict of interest was reported by the authors.

\section{References}

Baghban, M., \& Ayani, M. B. (2017). Estimation of surface heat flux in a one-dimensional hyperbolic bio-heat conduction problem with temperature-dependent properties during thermal therapy. Journal of the Brazilian Society of Mechanical Sciences and Engineering, 39(5), 1479-1489.

Crane, M., Hurley, N. J., Crane, L., Healy, A., Corrigan, O., Gallagher, K., \& McCarthy, L. (2004). Simulation of the USP drug delivery problem using CFD: Experimental, numerical and mathematical aspects. Simulation Modelling Practice and Theory, 12(2), 147-158.

Cregg, P., Murphy, K., \& Mardinoglu, A. (2012). Inclusion of interactions in mathematical modelling of implant assisted magnetic drug targeting. Applied Mathematical Modelling, 36(1), 1-34.

Haik, Y., Chen, C.-J., \& Chatterjee, J. (2002). Numerical simulation of biomagnetic fluid in a channel with thrombus. Journal of Information Visualization, 5(2), 187-195.

Haik, Y., Pai, V., \& Chen, C. (1999b). Biomagnetic fluid dynamics. In Fluid dynamics at interfaces (pp. 439-452).

Haik, Y., Pai, V., \& Chen, C.-J. (1999a). Development of magnetic device for cell separation. Journal of Magnetism and Magnetic Materials, 194(1), 254-261.

Henry, S. L., Concannon, M. J., \& Yee, G. J. (2008). The effect of magnetic fields on wound healing: Experimental study and review of the literature. Eplasty, 8, e40.

Higashi, T., Ashida, N., \& Takeuchi, T. (1997). Orientation of blood cells in static magnetic field. Physica B: Condensed Matter, 237, 616-620.

Higashi, T., Yamagishi, A., Takeuchi, T., Kawaguchi, N., Sagawa, S., Onishi, S., \& Date, M. (1993). Orientation of erythrocytes in a strong static magnetic field. Blood, 82(4), $1328-1334$.

Iino, M. (1997). Effects of a homogeneous magnetic field on erythrocyte sedimentation and aggregation. Bioelectromagnetics, 18(3), 215-222.

Khashan, S. A., \& Haik, Y. (2006). Numerical simulation of biomagnetic fluid downstream an eccentric stenotic orifice. Physics of Fluids, 18(p), 113601. 
Lahiri, B., Ranoo, S., \& Philip, J. (2017). Magnetic hyperthermia study in water based magnetic fluids containing TMAOH coated Fe $3 \mathrm{O} 4$ using infrared thermography. Infrared Physics and Technology, 80, 71-82.

Liangruksa, M., Ganguly, R., \& Puri, I. K. (2011). Parametric investigation of heating due to magnetic fluid hyperthermia in a tumor with blood perfusion. Journal of Magnetism and Magnetic Materials, 323(6), 708-716.

Lu, Y.-J., Wei, K.-C., Ma, -C.-C. M., Yang, S.-Y., \& Chen, J.-P. (2012). Dual targeted delivery of doxorubicin to cancer cells using folate-conjugated magnetic multi-walled carbon nanotubes. Colloids and Surfaces B: Biointerfaces, 89, 1-9.

Miltenyi, S., Müller, W., Weichel, W., \& Radbruch, A. (1990). High gradient magnetic cell separation with MACS. Cytometry, 11(2), 231-238.

Motta, M., Haik, Y., Gandhari, A., \& Chen, C.-J. (1998). High magnetic field effects on human deoxygenated hemoglobin light absorption. Bioelectrochemistry and Bioenergetics, 47(2), 297-300.

Mousavi, S. M., Farhadi, M., \& Sedighi, K. (2016). Effect of non-uniform magnetic field on biomagnetic fluid flow in a 3D channel. Applied Mathematical Modelling, 40(15), 7336-7348.

Mustapha, N., Amin, N., Chakravarty, S., \& Mandal, P. K. (2009). Unsteady magnetohydrodynamic blood flow through irregular multi-stenosed arteries. Computers in Biology and Medicine, 39(10), 896-906.

Nursal, T. Z., Bal, N., Anarat, R., Colakoglu, T., Noyan, T., Moray, G., \& Haberal, M. (2006). Effects of a static magnetic field on wound healing: Results in experimental rat colon anastomoses. The American Journal of Surgery, 192(1), 76-81.

Rosensweig, R. E. (1985). Ferrohydrodynamics. Cambridge: Cambridge University Press.

Sankar, D., \& Lee, U. (2011). FDM analysis for MHD flow of a non-Newtonian fluid for blood flow in stenosed arteries. Journal of Mechanical Science and Technology, 25(10), 2573-2581.

Schenck, J. F. (1996). The role of magnetic susceptibility in magnetic resonance imaging: MRI magnetic compatibility of the first and second kinds. Medical Physics, 23, 815.

Shercliff, J. A. (1965). Textbook of magnetohydrodynamics. Pergamon Press.

Shiga, T., Okazaki, M., Seiyama, A., \& Maeda, N. (1993). Paramagnetic attraction of erythrocyte flow due to an inhomogeneous magnetic field. Bioelectrochemistry and Bioenergetics, 30, 181-188.

Takeuchi, T., Mizuno, T., Higashi, T., Yamagishi, A., \& Date, M. (1995). Orientation of red blood cells in high magnetic field. Journal of Magnetism and Magnetic Materials, 140, $1462-1463$.

Tzirtzilakis, E. (2005). A mathematical model for blood flow in magnetic field. Physics of Fluids, 17, 077103.

Tzirtzilakis, E. (2008). Biomagnetic fluid flow in a channel with stenosis. Physica D: Nonlinear Phenomena, 237(1), 66-81.

Yamagashi, A., Takeuchi, T., Hagashi, T., \& Date, M. (1992). Diamagnetic orientation of blood cells in high magnetic field. Physica B: Condensed Matter, 177(1), 523-526.

Yellen, B. B., Forbes, Z. G., Halverson, D. S., Fridman, G., Barbee, K. A., Chorny, M., ... Friedman, G. (2005). Targeted drug delivery to magnetic implants for therapeutic applications. Journal of Magnetism and Magnetic Materials, 293(1), 647-654.

Yu, R., Chen, H., Chen, T., \& Zhou, X. (2008). Modeling and simulation of drug release from multi-layer biodegradable polymer microstructure in three dimensions. Simulation Modelling Practice and Theory, 16(1), 15-25. 